\title{
Effect of successive lists on the parameters of a paired-associate model
}

JOHN H. MUELLER, SAINT LOUIS UNIVERSITY

IRWIN D. NAHINSKY, UNIVERSITY OF MISSOURI

Each of $16 \mathrm{Ss}$ was required to learn four of $16 \mathrm{PA}$ lists differing in the number of stimuli and response alternatives. An effect on the parameters of a duoprocess learning model was found for the number of stimuli, but not for response alternatives, or for successive lists.

All-or-none mathematical models have typically been unconcerned with the influence of task variables, focusing on the learning process itself. However, recent studies have indicated that certain task parameters may influence the constants in paired-associate (PA) models, e.g., number of stimuli and response alternatives (Nahinsky \& Dodd, 1965), and training procedures (Bower, 1962).

The particular model of interest in the present investigation is that of Nahinsky $(1964,1967)$ which postulates an all-or-none association process when a correct response occurs, with the learning rate constantc, and an error elimination process when an incorrect response occurs, with the elimination rate constant a. Such a model is called duoprocess (DP), contrasted to a uniprocess (UP) model (e.g., Bower, 1961) which would involve only a single constant, very much like the DP case where $a=0$. The literature indicates that the UP model fares well for correction procedures (Bower, 1962), while the DP model provides a fit to noncorrection PA procedures (Nahinsky \& Dodd, 1965).

The present study is concerned with the DP model in a noncorrection PA task with the number of stimuli and response alternatives varied between lists and with Ss required to learn multiple lists. This will further clarify the effect of the number of stimuli, and determine whether practice on the task influences these parameters. Method

Subjects . Sixteen Ss were recruited from summer session psychology courses at Saint Louis University. No $S$ had previously served in a verbal learning experiment. Four Ss were assigned at random to each of four Latin squares.

Apparatuis . A Carousel 800 slide projector was used to present the PA lists. It was modified so that a bank of photocells read holes punched in the bottom of the slides. These holes corresponded in binary fashion to the correct numerical response. A panel with numbered switches was used by $S$ to indicate his response, and the bank circuitry then activated a "right" or "wrong" light to inform $\mathrm{S}$. The responding was self-paced, but the response activated a timer which presented the next slide 5 sec later. Response sequences were recorded with an Esterline Angus event recorder
Procedure and Design. Sixteen PA lists were used, with CVCs as the stimuli and the integers 1 to 8 as responses. Average list $M$ varied from $22 \%$ to $25 \%$ (Archer, 1960). Four lists had two stimuli with either two, four, six, or eight possible response alternatives (RA), with four other lists each for four, six, and eight stimuli. Primary similarity between lists was minimal. Randomization of presentation was used on successive trials, and two successive correct trials served as a criterion.

The design used four 4 by 4 Latin squares. In a single square, columns indicated list number, while the rows were different Ss, each learning the four lists in a different order. Each Slearned the four lists with the same number of RA, so that, for $\mathrm{k}$ RA, a given $S$ learned the $2 \mathrm{k}, 4 \mathrm{k}, 6 \mathrm{k}, 8 \mathrm{k}$ lists, with the first number indicating the number of stimuli and $k$ the number of RA. The number of RA was constant within a square, but varied between squares, $\mathrm{k}$ being either, $2,4,6$, or 8 . Thus Ss in the same square differed only on order of list learning and squares on RA number. The orders used within each square were as follows. Row $1: 2 \mathrm{k}, 4 \mathrm{k}, 6 \mathrm{k}$, $8 \mathrm{k}$; Row 2: $4 \mathrm{k}, 6 \mathrm{k}, 8 \mathrm{k}, 2 \mathrm{k}$; Row 3: 6k, 8k, 2k, 4k; Row $4: 8 k, 2 k, 4 k, 6 k$. These orders were used within each of the four squares.

\section{Results and Discussion}

Latin square analyses were performed for the separate squares and pooled squares, using the following measures as the data: trials to criterion, total errors, number of unlearned association trials (last errortrial plus one), chance correct, the empirical learning rate constant $(E C=$ number of stimuli/chance correct), and the DP predicted constant $(\mathrm{DPC}=$ number of $\mathrm{RA}$ minus one/twice the mean errors per stimulus). For the pooled analyses (Winer, 1962; Plan 9 with $n=1$ ), all six measures indicated a significant $(p<0.05)$ effect for stimuli number, while the column (list number) effects were all nonsignificant. The Squares (RA level) effect was significant only for errors and trials to criterion. All interactions of Squares with Stimuli and Lists were nonsignificant.

For Ec, the Lists effect for each RA level and pooled levels gave $F$ values less than 1.0. The Stimuli effect reached significance for the two RA square only ( $F=$ 6.05 , $\mathrm{df}=3 / 6)$, and for the pooled analysis $(\mathrm{F}=5.89, \mathrm{df}=$ $3 / 24)$. The Squares effect with Ec for the pooled analysis was also nonsignificant as noted above $(\mathrm{F}=0.51$, $\mathrm{df}=$ $3 / 12)$. With DPc, the List effect for pooled squares was nonsignificant ( $\mathrm{F}=1.99, \mathrm{df}=3 / 24)$, with no differences in the separate squares (all $F$ values less than 2.30 , $\mathrm{df}=$ $3 / 6)$. The pooled stimuli effect for DPc was significant 
Table 1. Learning Rate Constants and their Difference from the Empirical Constant.

Number of Stimuli

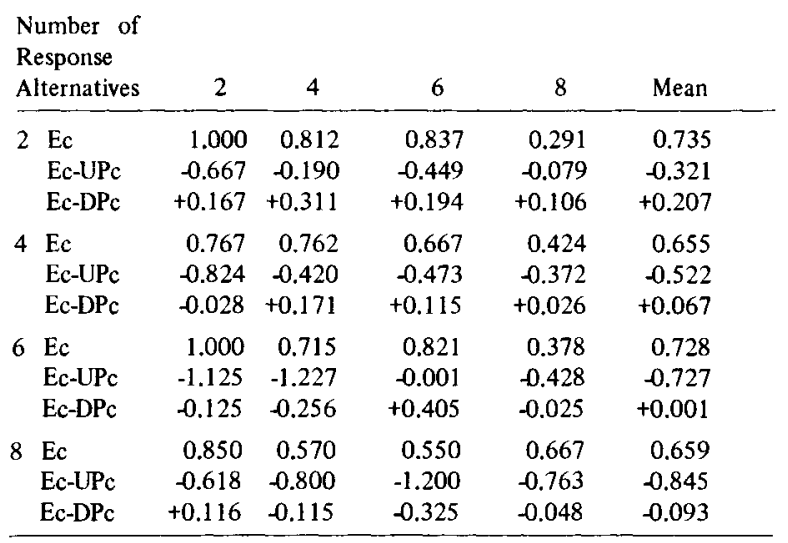

$(F=5.28, \mathrm{df}=3 / 24)$, but failed to reach significance in the individual RA levels, although the two and six RA squares produced moderate effects $(F=3.98$ and $F=2.51$, $d f=3 / 6$ ). The Squares main effect for DPc was nonsignificant $(F=1.13$, df $=3 / 12)$.

Therefore, the pooled analyses on Ec and DPc indicate that stimuli number affects the learning rate constants, but the number of RA and multiple lists do not. There was some deviation from this result for the individual squares, since the error terms for these are based on a very small number of degrees of freedom. While such a finding to some extent rules out nonspecific transfer as a source of variance within the limits of the materials used, no conclusion is possible for the effects of specific paradigmatic transfer. Since the practice effect seems limited, a series of observations might be taken on a $\mathrm{S}$ for use in determining his learning rate constant on other tasks of the same dimensions but differing in the specific content. This would avoid the necessity of recomputing these constants for the same $S$, adding to the generality of the model. The direction of change over the four lists was a slight increase, reaching significance only for the two RA case with Ec.
The present data also indicate a better fit for the DP model than for the UP model. Rigorous tests between models are not available, but the differences between the predicted UP or DP constants and the empirical constant indicate that only the two RA groups are closer to UPC than DPC. These differences are presented in Table 1 for the symmetrical DP model $(c=a$, UPc $=$ 2DPc). Since the two RA cases are essentially correction procedures, such a result might be expected. Analyses such as those described previously indicated that neither of these differences was significant. Since Ec tends to over-estimate due to correct guessing past the apparent learning point, the DP fit is actually somewhat better than indicated. It is also to be expected that asymmetrical DP values (Nahinsky, 1967) would provide closer approximations.

Vincentized stationarity analyses indicated that DP predictions were confirmed. Correct response probability on the second half of Ss'prelearning performance per pair was consistently higher than the first half, except in the two RA cases. Such an increase is expected by the DP model due to error elimination, but not by the UP model.

\section{References}

ARCHER, E. J. A re-evaluation of the meaningfulness of all possible CVC trigrams. Psychol. Monogr., 1960, 74, No. 10, Whole No. 497.

BOWER, G. H. Application of a model to paired-associate learning. Psychometrika, 1961, 26, 255-280.

BOWER, G. H An association model for responses and training variables in paired-associate learning. Psychol. Rev., 1962, 69, 3453.

NAHINSKY, I. D. A duoprocess model for paired associate learning. Psychol. Rep., 1964, 14, 467-471.

NAHINSKY, I. D. Statistics and moments parameter estimates for a duoprocess paired-associate model. J. Math. Psychol, 1967, 3, 140-150.

NAHINSKY, I. D., \& DODD, D. H. Learning rate constant in relationship to the number of stimuli and number of alternatives. Psychol Rep., 1965, 16, 1259-1260.

WINER, B. J. Statistical Principles in Experimental Design. New York: McGraw-Hill, 1962.

\section{Note}

1. This research was supported in part by NASA Training Grant NsG

(T) - 74, Supplement No. 3, awarded to the first author. 